\title{
HERRAMIENTAS INFORMÁTICAS DE GESTIÓN DE TERRENOS AGRARIOS: POTENCIALIDADES DEL MODELO AnnAGNPS EN NAVARRA
}

\author{
R. GIMÉNEZ* , J. CASALÍ, Y. CHAHOR \\ Universidad Pública de Navarra, E.T.S. de Ingenieros Agrónomos, \\ Departamento de Proyectos e Ingeniería Rural.
}

\begin{abstract}
RESUMEN. La actividad agraria está ampliamente afectada por las fluctuantes condiciones socio-económicas de la zona donde se enmarca. Es así que, una adecuada gestión de terrenos agrarios debería prever potenciales cambios en el uso de la tierra y las consecuencias de diversa índole derivadas de los mismos. Para ello, los modelos hidrológicos de simulación son una valiosa ayuda

En Navarra, el modelo AnnAGNPS (Annualized Agricultural Non-Point Source Pollution Model) ha sido exhaustivamente evaluado, concretamente en los buenos secanos cerealeros, obteniéndose resultados satisfactorios. En este trabajo se dan los rudimentos de la posible aplicación de este modelo en la gestión de la actividad agraria en territorio navarro.

En una pequeña cuenca cerealista se recrean distintos escenarios, con cultivos alternativos al actual. En cada caso, se analiza el efecto de estos cambios en la generación-promedio anual-de escorrentía y sedimentos, a la salida de la cuenca.

El modelo AnnAGNPS se presenta como una promisoria herramienta de gestión de tierras agrarias en ambientes mediterráneos.
\end{abstract}

Computer tools for agricultural land management: potentials of the AnnAGNPS model in Navarra

ABSTRACT. The Agricultural activity is largely affected by the fluctuating socioeconomic conditions of the region. Thus, an appropriate management of any agricultural land should anticipate potential changes in the land use and their consequences. For this, hydrological models are valuable aids.

In Navarre, AnnAGNPS (Annualized Agricultural Non-Point Source Pollution Model) has been extensively evaluated, specifically in dry lands devoted to cereals. In sums, satisfactory results were obtained. In this paper the rudiment of the possible application of this model in the management of the agricultural activity in Navarre is given. 
In a small grain-sown watershed, different scenarios with alternative potential crops are recreated by the model. In each case, the effect of these changes on the runoff and sediment generation-average annual values-at the watershed outlet are estimated.

Overall, AnnAGNPS appears as a promising tool for assessing the effect of the agricultural activity on the environment in the long run; and then for helping implementing adequate land management practices.

Palabras clave: modelos hidrológicos, AnnAGPNS, gestión territorial, escorrentía, sedimentos, región mediterránea, uso del suelo, Navarra.

Key words: hydrological models, AnnAGPNS, land management, runoff, sediment, Mediterranean region, land use, Navarre.

Enviado el 2 de septiembre de 2011 Aceptado el 30 de noviembre de 2011

* Correspondencia: Departamento de Proyectos e Ingeniería Rural. Universidad Pública de Navarra. Edificio Departamental de los Olivos, 31006-Pamplona (España). E-mail: rafael.gimenez@unavarra.es

\section{Introducción}

El estudio de los procesos erosivos no resulta tarea fácil dado el número de variables involucradas y su interacción, especialmente cuando el enfoque se realiza a macroescala (Giménez et al., 2012a, b). Así, a nivel de cuenca, la relación entre la descarga y la producción de sedimentos cambiará según las características climáticas (Vega et al., 1998; Singh et al., 2004); pudiendo presentar, incluso, importantes variaciones estacionales (p.ej., Gregory y Walling, 1973; Van Dijk y Kwaad, 1996; Casalí et al., 2008, 2010). Más aún, la salida de sedimentos en una cuenca estará también ampliamente determinada por las características de los diferentes eventos de lluvia y escorrentía, por las condiciones superficiales (Steegen et al., 2000) y contenido de humedad antecedente (Seeger et al., 2004) de los principales suelos, por el tipo de proceso erosivo dominante (ej., erosión laminar o por cárcava) (Regües et al., 2000), etc.

Con todo, la existencia de una cubierta vegetal -natural o debida a cultivos- es frecuentemente el principal determinante de los procesos hidrológicos-erosivos (García Ruiz y López Bermúdez, 2009); siendo el tipo de uso y manejo del suelo, por tanto, un factor decisivo a la hora de explicar dichos procesos.

En general, los principales terrenos de cultivos españoles presentan importantes problemas erosivos en cuanto aumenta la pendiente, ya que los suelos permanecen más o menos desprotegidos durante buena parte del año (García Ruiz y López Bermúdez, 2009). En concreto, la agricultura cerealista -especialmente en laderas empinadas- produce tasas muy altas de erosión (p. ej., Casalí et al., 2008; Giménez et al., 2012a); sobre 
todo si se alterna con barbechos. Por otro lado, en ambientes forestales, la escorrentía suele verse reducida como consecuencia de la interceptación de la lluvia y alto consumo de agua por parte del arbolado (García Ruiz y López Bermúdez, 2009). Es así que la producción de sedimentos en cuencas forestales suele ser relativamente baja y limitada a reducidos espacios dentro de las mismas. Igualmente, los pastos e incluso una cubierta densa de matorral pueden ser tan eficaces en reducir la tasa de erosión como lo sería el bosque. Aún así, un inadecuado manejo forestal puede dar lugar a pérdidas de suelo elevadas; aún superiores, incluso, a las registradas en terrenos cultivados con cereales (Casalí et al., 2010).

Las características ambientales mediterráneas -p.ej. irregularidad e intensidad de las precipitaciones, terrenos empinados, suelos vulnerables- crean condiciones propicias para la ocurrencia de intensos procesos erosivos; con los consecuentes perjuicios en el medio ambiente. Cabe destacar que el impacto ambiental del fenómeno erosivo no se limita a la disminución de la capacidad productiva del suelo y de transitabilidad de los terrenos afectados, sino también al efecto a distancia provocado por la posterior deposición del material erosionado. Así, los quebrantos económicos causados por la colmatación y/o polución de lagos, ríos y embalses muchas veces superan a los producidos en el terreno.

En Navarra, en particular, la erosión de suelos agrícolas es -igualmente- un problema de significativa magnitud (Casalí, 1999; De Santisteban et al., 2006; García Ruiz y López Bermúdez, 2009). Precisamente, y con el propósito de valorar el efecto de la actividad agraria sobre el entorno y contemplar mejoras en el manejo de las zonas afectadas, el Gobierno de Navarra mantiene -desde hace ya varios años- diversas cuencas agrarias, típicas de su territorio. De éstas se recoge, en forma continua, información meteorológica, de generación de sedimentos y de calidad de aguas (Giménez et al., 2012a, b). Todo esto permite, a largo plazo, evaluar cabalmente el impacto del uso actual de la tierra.

Sin embargo, una adecuada gestión de terrenos agrarios, debería contemplar la posibilidad de cambios en el uso de la tierra; ya sea para buscar mejoras en el manejo de las zonas afectadas o bien para adecuarse a nuevas políticas socio-económicas. Para tal previsión, los modelos hidrológicos resultan valiosas herramientas que permiten evaluar principalmente el efecto medioambiental de escenarios (agrarios) ficticios; lográndose esto con una -relativamente- baja inversión de tiempo y dinero.

De hecho, existen diversos modelos hidrológicos-erosivos que varían, principalmente, en su complejidad matemática, en los procesos simulados y en el tipo de datos requeridos para su calibración y posterior validación. Ejemplos de éstos son ANSWERS (Bouraoui et al., 2000); EUROSEM (Morgan, 1998), SWAT (Arnold et al., 1998), MIKE SHE (Christiaens y Feyen, 2002) y -entre los más destacados- AnnAGNPS (Bigner et al., 2003). Una descripción detallada de los principales modelos se encuentra en Borah y Bera (2003).

No obstante, no existe -al menos a nivel de cuenca- un único modelo de aplicación universal; el más idóneo dependerá, mayormente, del tipo de proceso(s) a evaluar y de las características y condiciones del medio. Aún más, la fehaciente aplicación de un determi- 
nado modelo requerirá de una exhaustiva -y a menudo, laboriosa- puesta a punto (calibración y validación); con el fin de buscar el mejor ajuste a las condiciones del medio natural.

Precisamente, diversos investigadores - a nivel mundial- han evaluado la capacidad del citado modelo AnnAGNPS en predecir escorrentía y producción de sedimentos, bajo diferentes condiciones climáticas y de usos del suelo. Aunque, son todavía pocos los estudios llevados a cabo en ambientes mediterráneos (tabla 1).

De ahí que la capacidad predictiva de AnnAGPNS - en cuanto a escorrentía y producción de sedimentos y nitratos- fue recientemente evaluada en una pequeña cuenca cerealista (Laxtaga) de Navarra a lo largo de 6 años (2003-2008) de registro continuo de datos. Los resultados indican una adecuada aplicabilidad del modelo a las condiciones locales (Chahor et al., en preparación); asumiéndose, además, una buena capacidad del mismo para mantener resultados fidedignos frente a potenciales cambios en el uso y manejo del suelo.

En este trabajo se realiza un ejercicio de simulación -usando el modelo AnnAGNPSde la escorrentía y sedimentos generados a la salida de una pequeña cuenca agraria de Navarra, frente a potenciales cambios en el uso y manejo de la misma.

No se pretende más que vislumbrar las potencialidades de AnnAGNPS como herramienta de gestión de terrenos agrarios de Navarra. Cabe destacar que esta aplicación -sólo en apariencia simple- resultaría dudosa y cuestionable sin la concienzuda evaluación previa del modelo para nuestras condiciones; apoyada, por cierto, en una larga y detallada base de datos propia.

\section{Materiales y métodos}

\subsection{Breve descripción del modelo AnnAGNPS}

El Departamento de Agricultura de Estados Unidos de América desarrolló, para la gestión ambiental, el modelo AnnAGNPS -Annualized Agricultural Nonpoint Source Pollution- (Bingner et al., 2003). Es éste un modelo concebido para simular el transporte de agua, sedimentos y químicos a nivel de cuenca, permitiendo, así, evaluar el impacto ambiental de potenciales actividades agrícolas y prácticas de manejo de suelos.

Se trata de un modelo semi-empírico, pero con módulos de base física, distribuido -la cuenca en estudio puede dividirse en diferentes zonas, susceptibles de calibrarse de manera independiente-, continuo, y de paso diario.

En AnnAGNPS la cuenca es dividida en celdas que, al ser definidas siguiendo criterios topográficos, resultan normalmente de forma irregular. Estas celdas se ajustan a un diseño espacial tal que todas ellas confluyen, engarzadas, a la red de drenaje. Asimismo, cada celda presenta un único valor tanto de tipo de suelo, como de uso de la tierra y manejo. Para ello, si al definirse una celda cualquiera quedara involucrado, por ejemplo, más de un tipo de suelo, el modelo consideraría como único suelo, al predominante; y así para todas las variables implicadas. 


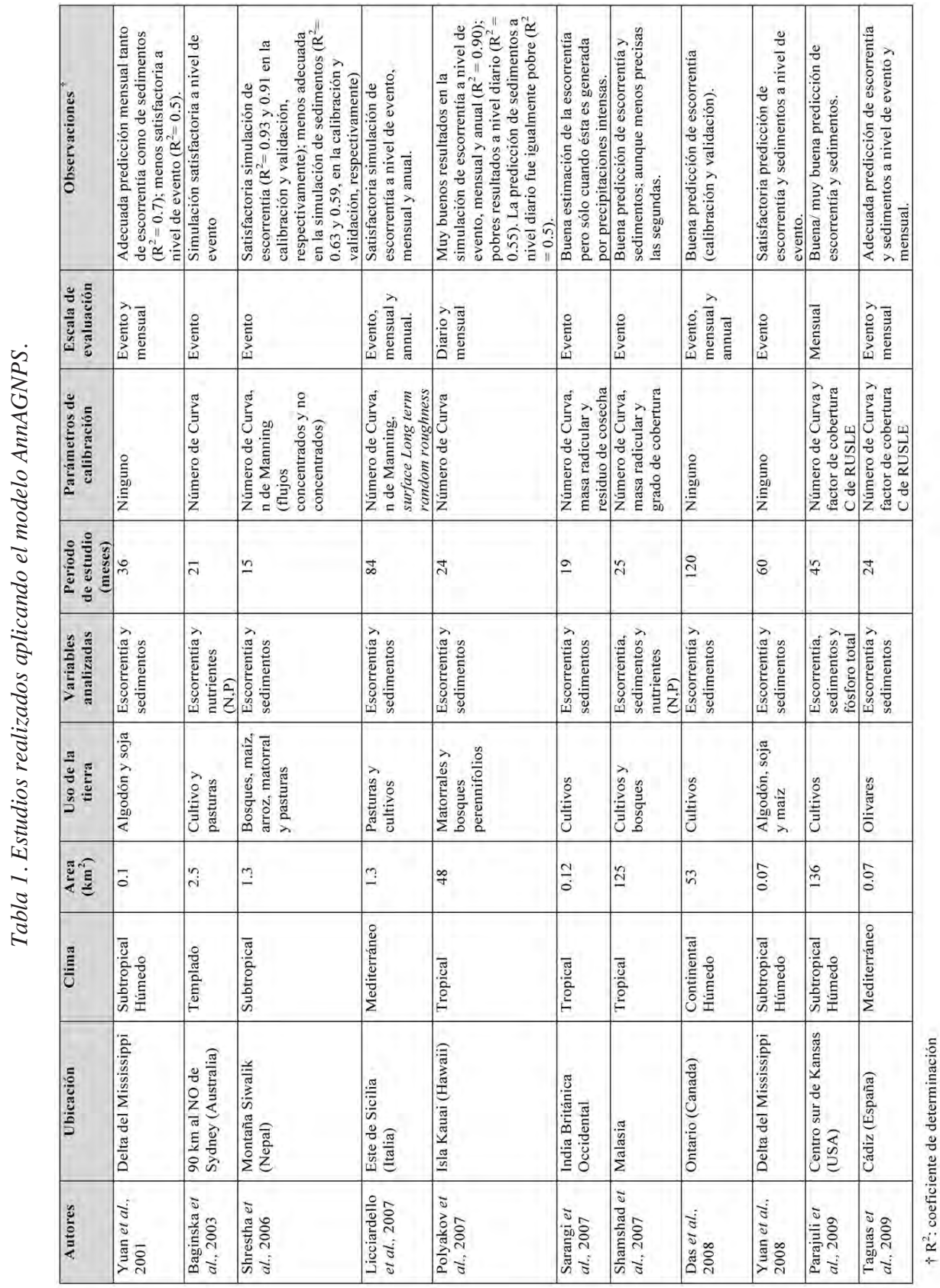


Básicamente, AnnAGNPS requiere dos clases de datos de entrada. Primero, datos climáticos, discriminados en forma diaria (p.ej., precipitación, temperatura del aire, dirección y velocidad del viento). Y en segundo término, información sobre características físicas de la cuenca (p.ej., parámetro morfológicos, información sobre suelos, cultivos, prácticas agrícolas).

El modelo utiliza datos climatológicos diarios para realizar un balance de agua en el suelo y estimar el Número de Curva (SCS, 1986) -dependiente éste, precisamente, del contenido de humedad edáfica-. La producción de escorrentía se determina a partir del método del citado Número de Curva, pero parcialmente modificado. Asimismo, las pérdidas de suelos en cada celda se estiman a través del conocido modelo RUSLE - Revised Universal Soil Loss Equation- (Renard et al., 1997). Por otro lado, la descarga de sedimentos a la red de drenaje se determina mediante el modelo HUSLE -Hydro-geomorphic Universal Soil Loss Equation-(Theurer y Clarke, 1991).

Finalmente, los valores de salida del modelo pueden ser definidos a diferentes escalas temporales: diaria, mensual o anual.

\subsection{Caso de estudio: cuenca experimental Latxaga}

La cuenca de Latxaga (207 ha) se ubica en el centro-este de Navarra en terrenos de los términos municipales de Lizoain y Urroz-Villa (fig. 1).

Domina un clima sub-mediterráneo húmedo, con una precipitación anual media de $835 \mathrm{~mm}$ y una temperatura media de $12^{\circ} \mathrm{C}$ (Gobierno de Navarra, 2001).

Los fondos de valle son de pendiente suave (5-7\%), pero con laderas empinadas (hasta 30\%). Los suelos predominantes son Paralitic Xerorthent y Fluventic Haloxerept (USDA, Soil Taxonomy, 2006). Una completa descripción de la cuenca se detalla en Giménez et al. (2012a); como así también, la respuesta hidrológica y erosiva de la misma a lo largo de más de una década de actividad agraria.

En cuanto al uso y manejo del suelo, la cuenca se encuentra ocupada prácticamente en su totalidad (85-90\%) con cereales de invierno -trigo, cebada y avena-; por cierto, uso típico de la zona. Dispersas en toda la cuenca se observan, además, pequeñas áreas improductivas -bajo matorral-, constituyendo aproximadamente un $10 \%$ de la superficie total. Asimismo, una (densa) faja de vegetación natural es mantenida -ex profeso- a lo largo de los principales cauces. Por otro lado, se practica un laboreo convencional, normalmente siguiendo curvas de nivel.

\subsection{Potenciales escenarios agrícolas}

A lo largo de la historia de España, el tipo de explotación de un mismo territorio ha sufrido cambios -normalmente lentos- inducidos por fenómenos culturales, demográficos o bien económicos. En las últimas décadas, estos cambios -ahora, generalmente ace- 

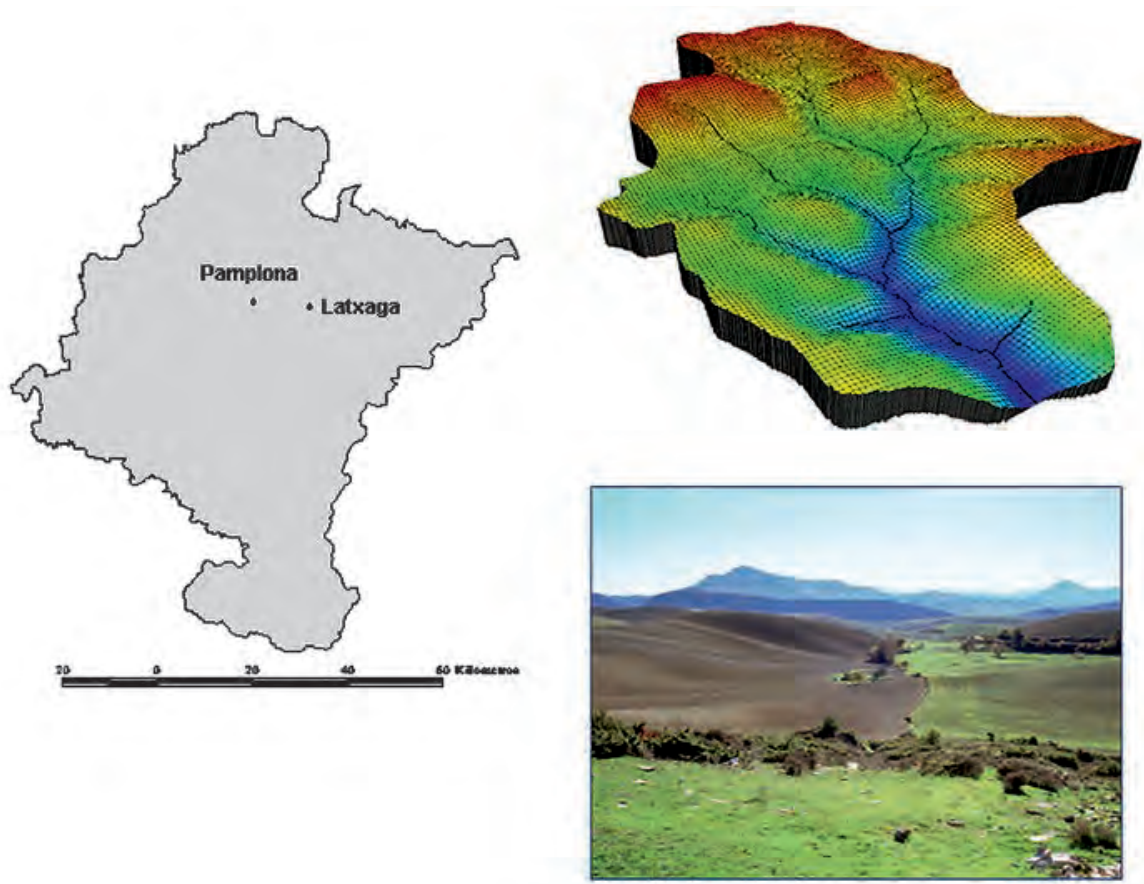

Figura 1. Ubicación de la cuenca experimental de Latxaga.

lerados- fueron inducidos por el mercado y/o por la Política Agraria Comunitaria (PAC). Es así que el mercado globalizado ha favorecido -principalmente a través de subvenciones- la rápida expansión de determinados sistemas de explotación y cultivos en detrimento de otros usos de la tierra. Incluso se ha llegado a incentivar el abandono de tierras (set-aside) para favorecer la conservación del suelo y mejorar su balance hídrico (García Ruiz y López Bermúdez, 2009).

En Navarra, los cereales - principalmente, cebada y trigo- representan, desde hace tiempo, aproximadamente el $80 \%$ de los cultivos herbáceos; con una especial presencia en los secanos de la zona central del territorio, debido a óptimas condiciones ecológicas. Sin embargo, los principales usos de la tierra en la región de Navarra han sufrido también (importantes) cambios en las últimas décadas. Por ejemplo, la superficie bajo cultivo de colza a principio de los años 90 del siglo pasado quintuplicaba a la existente a fines de la década precedente; para decrecer drásticamente, sin embargo, pocos años después. Ciertamente, la presencia de este cultivo es, en la actualidad, poco más que testimonial, a pesar de la reciente llegada de la industria del biodiesel (Goñi et al., 2008). Igualmente, el cultivo del girasol experimentó un brusco incremento en la primera mitad de los noventa, seguido de un también rápido descenso; manteniéndose, de ahí en adelante, con valores oscilantes -aunque relativamente elevados-(Lezaun et al., 2004). De hecho, el girasol es actualmente una alternativa tradicional a los cereales en la Baja Montaña y Zona 
Media. Por otra parte, la superficie ocupada por guisante proteaginoso ha experimentado un manifiesto incremento a partir del año 1995; sobre todo durante los primeros años del presente siglo; esto gracias a incentivos económicos emanados de la PAC.

Por lo expuesto, resultaría debidamente fundada la propuesta de los siguientes escenarios concebidos como potenciales usos agrícolas en nuestra cuenca de estudio (Latxaga):

Escenario 1: $60 \%$ cereales, $25 \%$ girasol.

Escenario 2: $60 \%$ cereales, $25 \%$ colza.

Escenario 3: 60\% cereales, $25 \%$ leguminosas.

Escenario 4: $60 \%$ cereales, $25 \%$ (girasol + colza + leguminosas, en partes iguales).

Escenario 5: 60\% cereales y alternativamente, $20 \%, 30 \%, 40 \%$ y $50 \%$ de matorral.

Los valores porcentuales están referidos a la superficie total de la cuenca. Aproximadamente un $12 \%$ de la cuenca es, como ya se indicó, improductiva (suelos someros, afloramientos rocosos, construcciones). La presencia de matorral pretende reflejar la situación de tierras abandonadas (sin cultivar), ya que el matorral mediterráneo (tomillares, aliagares, romerales, etc.) constituye la principal vegetación natural de la zona (Departamento de Agricultura. Ganadería y Alimentación del Gobierno de Navarra).

Cabe aclarar que toda predicción a través de herramientas de simulación -sobre todo en ambientes complejos, como los nuestros- es, desde luego, incierta. Así, para reducir al mínimo esta incertidumbre es importante que los escenarios a ser recreados sean similares a aquellos en los cuales el modelo fue -en su momento- debidamente contrastado. De ahí que los escenarios propuestos no sólo son plausibles sino que, además, no se alejan en demasía de las condiciones reales bajo las cuales AnnAGNPS fue evaluado (ver abajo).

\section{Simulación con AnnAGNPS. Análisis de resultados}

La producción media de escorrentía y producción de sedimentos a la salida de la cuenca, en los diferentes escenarios, fueron estimados mediante la aplicación del modelo AnnAGNPS con datos meteorológicos e hidrológicos registrados en la cuenca de Latxaga entre 2003 y 2008, ambos inclusive (Chahor et al., en preparación). En la tabla 2 se indican los datos de manejo, propios de cada cultivo, usados en la simulación.

En un trabajo previo (Chahor et al., en preparación), la eficacia del modelo fue debidamente evaluada al simular - para el mismo período de tiempo- la escorrentía y exportación de sedimentos en las condiciones -a la sazón- actuales de uso de la tierra; contándose para ello con una extensa base de datos. Es así que, ajustando los valores iniciales -dados por defecto- del Número de Curva (CN) a los diferentes estados del cultivo, se logró una adecuada simulación de la escorrentía a escala anual; pero registrándose, aún así, una cierta sobreestimación durante la época seca. La descarga de sedimentos, por su parte, fue debidamente simulada -también a escala anual- tras el 
ajuste tanto del coeficiente de rugosidad de Manning como del coeficiente de rugosidad superficial aleatoria a largo plazo (surface long-term random roughness coefficient) (Chahor et al., en preparación).

Tabla 2. Cronograma de labores agrícolas en Latxaga aplicado en la simulación. Información recabada de entrevistas a agricultores de la zona.

\begin{tabular}{|c|c|c|c|}
\hline Cultivo & Fecha & Operación & Observación \\
\hline \multirow[t]{8}{*}{ Cereal/colza } & 15 Septiembre & Laboreo & Vertedera $(25 \mathrm{~cm})$ \\
\hline & 20 Septiembre & Cama de siembra & Rastra \\
\hline & 10 Octubre & Fertilización & \\
\hline & 15 Octubre & Siembra & Sembradora combinada \\
\hline & 15 Enero & Fertilización & \\
\hline & 15 Febrero & Desmalezado químico & \\
\hline & 15 Marzo & Fertilización & \\
\hline & 1 Julio & Cosecha & \\
\hline \multirow[t]{5}{*}{ Leguminosa } & 15 Septiembre & Laboreo & Vertedera $(25 \mathrm{~cm})$ \\
\hline & 20 Septiembre & Cama de siembra & Rastra \\
\hline & 1 Diciembre & Siembra & Sembradora \\
\hline & 3 Diciembre & Desmalezado químico & \\
\hline & 1 Julio & Cosecha & \\
\hline \multirow[t]{6}{*}{ Girasol } & 20 Septiembre & Laboreo & Vertedera $(25 \mathrm{~cm})$ \\
\hline & 15 Marzo & Cama de siembra & Rastra \\
\hline & 1 Mayo & Siembra & Sembradora de precisión \\
\hline & 3 Mayo & Desmalezado químico & \\
\hline & 15 Mayo & Fertilización & \\
\hline & 15 Julio & Cosecha & \\
\hline
\end{tabular}

No existiendo marcadas diferencias de fertilidad física (por ej. textura, profundidad) y química entre los suelos dominantes, los diferentes cultivos -que conforman cada escenario- fueron distribuidos aleatoriamente en la cuenca, hasta ocupar la totalidad de la superficie prefijada. Bajo otras circunstancias, se habría procedido, por ejemplo, a ubicar el girasol en los suelos más profundos y fértiles y la colza en suelos de texturas francas que permitieran una rápida nacencia (Alberto Lafarga, comunicación personal).

Cabe destacar que si relacionamos directamente la exportación de sedimentos con la intensidad de pérdida del suelo, estaríamos cometiendo una cierta subestimación ya que parte del suelo erosionado podría haberse depositado antes de alcanzar la salida de la cuenca. Sin embargo, en cuencas pequeñas -como la presente- podemos asumir que dicha deposición es mínima. 

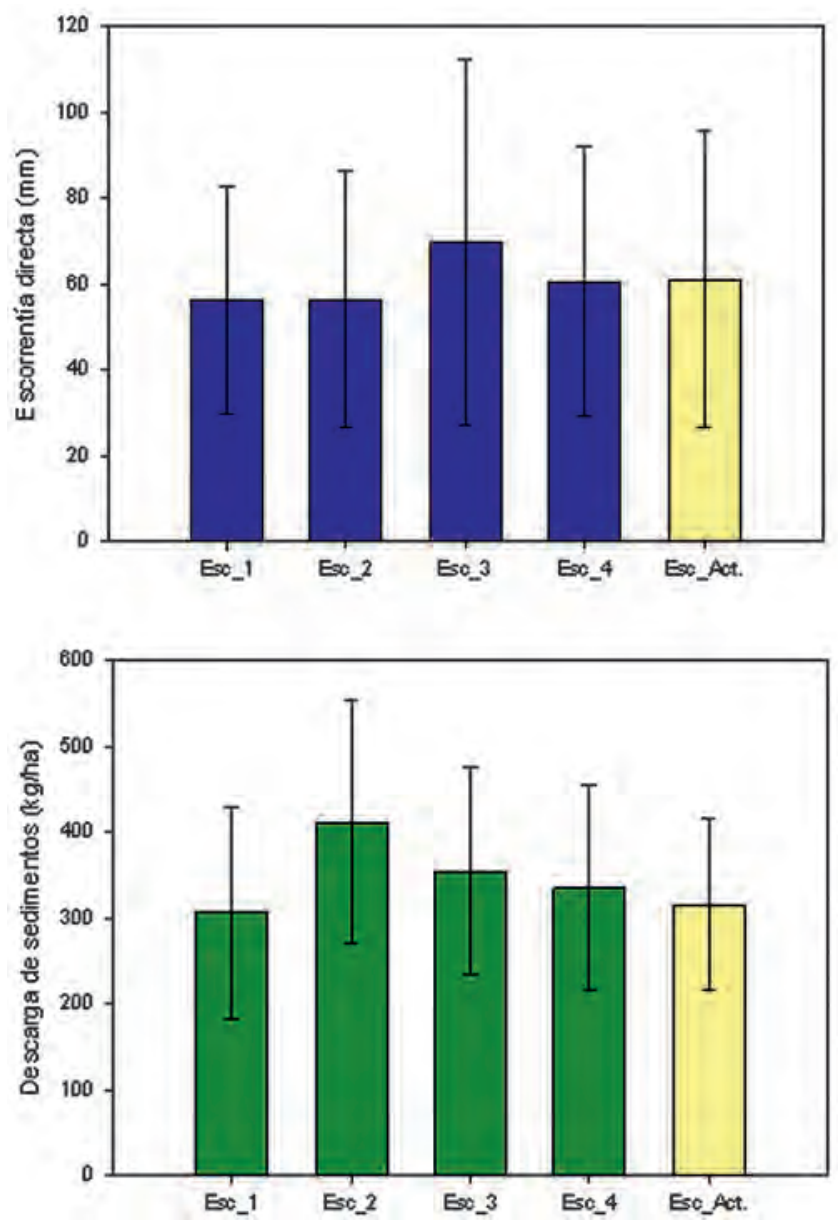

Figura 2. Volumen medio anual de escorrentía directa simulado por AnnAGNPS en los distintos escenarios ficticios (ver texto por detalles). Esc. Act. corresponde a la simulación bajo las condiciones actuales de uso de la tierra.

Destacamos que la condición de "matorral” se recreó en el modelo asumiendo una masa anual de raíces de $5000 \mathrm{~kg} / \mathrm{ha}$; un porcentaje de cobertura de $90 \%$ y un valor anual de altura de caída de lluvia de $0.2 \mathrm{~m}$.

Analizando en primer término la situación actual/real de la cuenca -Esc. Act, fig. 2-, los valores medios de escorrentía directa (descontado el flujo base) y de descarga de

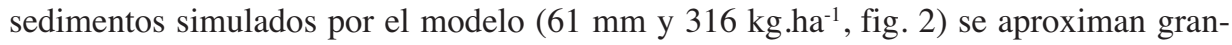
demente a los valores reales registrados en igual período (Chahor et al., en preparación): $57 \mathrm{~mm}$ y $309 \mathrm{~kg} \cdot \mathrm{ha}^{-1}$, respectivamente. Esta óptima simulación sugiere que los resultados a estimar en nuestros potenciales escenarios no se alejarían -llegado el caso- considerablemente de la realidad.

Ahora sí, con respecto a nuestros escenarios ficticios, no se observan significativas diferencias en cuanto a generación de escorrentía, salvo un ligero aumento cuando el 
cultivo alternativo es leguminosa. Sin embargo, sí se observan mayores contrastes en cuando a la descarga media de sedimentos a la salida de la cuenca. Si bien el cultivo del girasol generaría similares pérdidas de suelos que las sufridas actualmente (fig. 2), los restantes cultivos alternativos provocarían cambios más notorios; especialmente el cultivo de colza que generaría un incremento en la producción de sedimentos de alrededor de un $30 \%$ (fig. 2). Evidentemente, reduciendo la superficie ocupada por colza a favor de otros cultivos (Esc. 4) (girasol y leguminosa), podríamos llegar a situaciones de exportación de sedimentos próximas a las actuales (fig. 2).

Siendo que las simulaciones fueron realizadas todas bajo similares condiciones -incluso se asumió para la colza, Esc 2, idéntico laboreo que para la situación actual, Esc.Act; tabla 2- estas diferencias se deberían principalmente al disímil grado de cobertura del suelo y de las características y permanencia del rastrojo (por ej., relación peso del residuo vegetal/peso de grano) propios de cada cultivo.

Con todo, estos incrementos porcentuales previstos de erosión del suelo para los escenarios ficticios no resultarían preocupantes ya que, actualmente, estos valores en la cuenca de Latxaga están muy por debajo de los umbrales de tolerancia (1.12 $\mathrm{kg} \mathrm{m}^{-2} \mathrm{año}^{-1}$; Hall et al., 1985). Sin embargo, si trasladásemos nuestros escenarios a cuencas cerealistas similares a Latxaga - p. ej. cuenca de La Tejería; Giménez et al., 2012a-, pero no obstante más vulnerables a la erosión -y por ende con una exportación media de sedimento muy superior a la de Latxaga-, estas estimaciones sí resultarían preocupantes.

En cuanto a la situación de abandono de tierras (presencia de matorral), se observa una clara tendencia decreciente de la producción de sedimentos (y de escorrentía) con el aumento de la superficie bajo matorral (fig 3). Cabe destacar que, si bien se predice -en realidad- un repunte de la escorrentía a partir de un $40 \%$ de retirada de tierra (fig. 3), éste no afectaría la mencionada menguante tendencia de los sedimentos. En general, se acepta que el abandono favorece una reducción de la erosión y de la escorrentía (García Ruiz y López Bermúdez, 2009), tal como se ha comprobado experimentalmente (p. ej., Lasanta et al., 2006). Sin embargo, esto es así a partir de estados avanzados de colonización por parte del matorral, momento en el cual se produce -efectivamente- una reducción en la generación de escorrentía y en la erosión. De hecho, tras los primeros meses de abandono -cuando el matorral está todavía poco desarrollado- se pueden alcanzar, por el contrario, elevadas tasas de erosión. Esto último explicaría la paradójica presencia de suelos deteriorados bajo densos matorrales.

\section{Conclusiones}

El modelo AnnAGNPS se presenta como una promisoria herramienta de gestión de tierras agrarias en ambientes mediterráneos. Sin embargo, su implementación normalmente demandará -como prácticamente la totalidad de los modelos, por cierto- una exhaustiva evaluación previa de su comportamiento bajo las condiciones reinantes; y posteriormente -de ser necesario- de un ajuste a las mismas. Así, la calibración del modelo para una aceptable predicción de la escorrentía y de la producción de sedimen- 

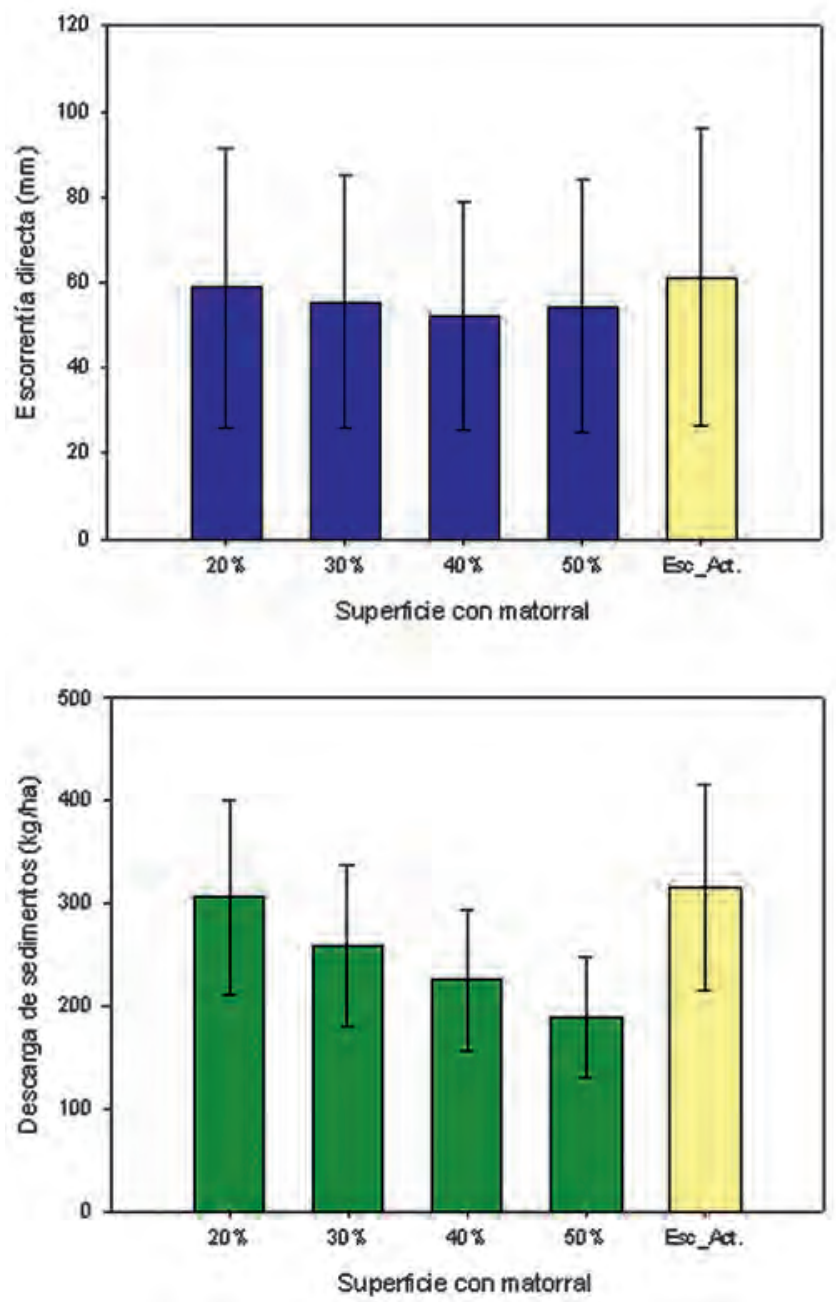

Figura 3. Descarga media anual de sedimentos simulada por AnnAGNPS en los distintos escenarios ficticios (ver texto por detalles). Esc. Act. corresponde a la simulación bajo las condiciones actuales de uso de la tierra.

tos -ambos, valores medios anuales- en secanos de Navarra va a requerir, mínimamente, modificar los valores -dados por defecto- del Número de Curva (escorrentía) y de los coeficientes de rugosidad superficial (sedimentos), entre otros.

Aunque no se haya ejemplificado en este trabajo, AnnAGNPS simula adecuadamente la generación y tránsito de (agro)químicos; permitiendo así predecir, por ejemplo, el impacto de la actividad agraria en la calidad del agua.

\section{Agradecimientos}

Estos estudios fueron parcialmente financiados a través de los proyectos REN200303028/HID, CGL2006-07236/CLI, CGL2007-63453/HID y CGL2011-24336 pertene- 
cientes a diferentes ministerios competentes en materia de investigación del Gobierno de España. Los autores agradecen, además, la generosa contribución del Departamento de Desarrollo Rural, Industria, Empleo y Medio Ambiente del Gobierno de Navarra, en especial de D. Joaquín del Valle de Lersundi Manso de Zúñiga. Agradecemos, asimismo, el asesoramiento recibo por Alberto Lafarga Arnal del Instituto Técnico y de Gestión Agrícola (ITGA) de Navarra.

\section{Referencias bibliográficas}

Arnold, J. G., Srinivasan, R., Muttiah, R. S., Williams, J. R. (1998). Large area hydrologic modelling and assessment part 1: Model. Paper No. 96089 of the Journal of the American Water Resources Association., 34 (1): 73-89.

Bingner, R. L., Theurer, F. D., YuAn, Y. (2003). AnnAGNPS Technical Processes. [http://www .ars.usda.gov/Research/docs.htm?docid=5199].

BorAh, D. K., BERA, M. (2003). Watershed-scale hydrologic and nonpoint source pollution models: Review of mathematical bases. Soil Water Div. ASAE, 46 (6): 15531566.

Bouraoui, F., Dillaha, T. A. (1996). Answers-2000: Runoff and sediment transport model Journal of Environmental Engineering, 122 (6): 493-501.

Casalí, J., LóPez, J. J., Giráldez, J. V. (1999). Ephemeral gully erosion in southern Navarra (Spain). Catena, 36: 65-84.

Casalí, J., Gastesi, R., Álvarez-Mozos, J., De Santisteban, L. M., Lersundi, J. D. V., Giménez, R., Larrañaga, A., Goñi, M., Agirre, U., CAmpo, M. A., LóPez, J. J., DONEZAR, M. (2008). Runoff, erosion, and water quality of agricultural watersheds in central Navarre (Spain). Agricultural Water Management, 95: 1111-1128.

Casalí, J., Giménez, R., Díez, J., Álvarez-Mozos, J., Del Valle de Lersundi, J., GoÑi, M., CAmpo, M. A., Chahor, Y., Gastesi, R., LóPez, J. (2010). Sediment production and water quality of watersheds with contrasting land use in Navarre (Spain). Agricultural Water Management, 97: 1683-1694.

Chahor, Y., Casalí, J., Giménez, R., Campo, M. A., Goñi, M. (en preparación). Evaluation of the AnnAGNPS model for predicting runoff and sediment yield in a small Mediterranean watershed, in Navarre (Spain).

Christiaens, K., Feyen J. (2002). Use of sensitivity and uncertainty measures in distributed hydrological modeling with an application to the MIKE-SHE model. Water Resource Research, 38: 81-815.

De Santisteban, L. M., Casalí, J., LóPez, J. J. (2006). Assessing soil erosion rates in cultivated areas of Navarre (Spain). Earth Surface Processes and Landforms, 31: 487-506.

García Ruiz, J. M., LóPez Bermúdez, F. (2009). La erosión del suelo en España. Sociedad Española de Geomorfología, 441 pp. 
GimÉnEZ, R., CASAlí, J., DíEZ, J. (2012a). Evaluación y producción de sedimentos y calidad de las aguas en cuencas agrarias de Navarra. Cuadernos de Investigación Geográfica, 38 (1): 7-25.

Giménez, R., Casalí, J., Grande, I., Díez, J., Campo, M. A., Álvarez-Mozos, J., GoÑI, M. (2012b). Factors controlling sediment export in a small agricultural watershed in Navarre (Spain). Agricultural Water Management, 110: 1-8.

Gobierno de Navarra (2001). Estudio Agroclimático de Navarra (CD). Gobierno de Navarra, Departamento de Agricultura, Ganadería y Alimentación. Servicio de Estructuras Agrarias. Pamplona. Spain.

Goñi, J., Irañeta, I., Sexmilo, J.,R., Lafarga. A. (2008). La colza en Navarra. Navarra Agraria. Septiembre-Octubre 2008. [http://www .navarraagraria.com/n170/ arcolz08.pdf].

Gregory, K. J., Walling, D. E. (1973). Drainage Basin Form and Process. A Geomorphological Approach. Edward Arnold, London.

Hall, G. F., Logan, T. J., Young, K. K. (1985). Criteria for determining tolerable erosion rates. En: Soil Erosion and Crop Productivity (Follett, R.F., Stewart, B.A. Eds.). American Society of Agronomy, Crop Science Society of America, Soil Science Society of America: Madison, Wisconsin.

Lasanta, T., Beguería, S., García Ruiz, J. M. (2006). Geomorphic and hydrological effects of traditional shifting agricultural in a Mediterranean mountain, Central Spanish Pyrenees. Mountain Research and Development, 26 (2): 146-152.

Lezaun, J. A., Armesto, A. P., Lafarga, A., GoÑI, J. (2004). Girasol. Navarra Agraria. Marzo-Abril 2004. [http://www.navarraagraria.com/n143/argira4.pdf].

Morgan, R. P. C., Quinton, J. N., Smith, R. E., Govers, G., Poesen, J. W. A., Auerswald, K., Chisci, G., Torri, D., Styczen, M. E. (1998). The European soil erosion model (EUROSEM): a dynamic approach for predicting sediment transport from fields and small catchments. Earth Surface Processes and Landforms, 23: 527-544.

Regües, D., Balasch, J. C., Castelltort, X., Soler, M., Gallart, F. (2000). Relación entre las tendencias temporales de producción y transporte de sedimentos y las condiciones climáticas en una pequeña cuenca de montaña mediterránea (Vallcebre, Pirineos Orientales) Cuadernos de Investigación Geográfica, 26: 41-65.

Renard, K. G., Foster, G. R., Weesies, G. A., McCool, D. K., Yoder, D. C. (coord.), (1997). Predicting Soil Erosion by Water: A Guide to Conservation Planning with the Revised Universal Soil Loss Equation (RUSLE). USDA Agriculture Handbook, 703.

SCS (1986). Technical Release 55: Urban hydrology for small watersheds. Soil Conservation Service, USDA.

Seeger, M., Errea, M. P., Begueria, S., Arnáez, J., Martí, C., García-Ruiz, J. M. (2004). Catchment soil moisture and rainfall characteristics as determinant factors 
for discharge/suspended sediment hysteretic loops in a small headwater catchment in the Spanish Pyrenees. Journal of Hydrology, 288: 299-311.

Singh, K. P., Malik, A., Mohan, D., Sinha, S. (2004). Multivariate statistical techniques for the evaluation of spatial and temporal variations in water quality of Gomti River (India). A case study. Water Research, 38 (18): 3980-3992.

SoHrABI, T. M. (2006). Uncertainty in TMDL models. Transactions of the ASAE, 49 (4): 1033-1049.

Steegen, A., Govers, G., Nachtergaele, J., Takken, I., Beuselinck, L., Poesen, J. (2000). Sediment export by water from an agricultural catchment in the Loam Belt of central Belgium. Geomorphology, 33 (1-2): 25-36.

Theurer, F. D., Clarke, C. D. (1991). Wash load component for sediment yield modeling. En Proceedings of the fifth federal interagency sedimentation conference: 7-1 to 7-8.

VAN DiJK, P. M., KwAAD, F. J. P. M. (1996). Runoff generation and soil erosion in small agricultural catchments with loess-derived soils. Hydrological Processes, 10 : 1049-1059.

Vega, M., Pardo, R., Barrado, E., Debán, L. (1998). Assessment of seasonal and polluting effects on the quality of river water by exploratory data analysis. Water Research, 32 (12): 3581-3592. 